\title{
Detección de lesiones oculares en niños seropositivos para Toxocara canis
}

\author{
Juan E. Sánchez T., Juan P. López G., Militza González N., Eduardo Villaseca D., \\ Denise Manieu M., Alejandra Roizen B., Isabel Noemí H. y Alejandro Viovy A.
}

\section{Prevalence of ocular lesions in children seropositive to Toxocara canis}

Intraocular invasion by Toxocara canis is one of the most commonly recognized etiologies of uveitis and blindness in children. In order to estimate the prevalence of ocular lesions caused by toxocariasis in a pediatric referral hospital, we conducted a retrospective analysis of clinical charts of all children seropositive to Toxocara canis, who also had an eye exam between the years 2005 and 2009 at the Calvo Mackenna Children's Hospital in Santiago, Chile. We identified 175 children [mean age 6 years (range 0.66-15)] seropositive to Toxocara canis, who had dilated eye exam. Only one child [(0.57\%); 95\% CI, - $0.55-1.69]$ had ophthalmoscopic findings compatible with Toxocara canis infection. The patient also suffered from decreased vision of the affected eye. The information gained from this study may be of useful for the implementation of algorithms for the ophthalmological examination of children seropositive to Toxocara canis in public hospitals in Chile.

Key words: Toxocariasis, ocular, prevalence, eye infections (parasitic).

Palabras clave: Toxocariosis, ocular, prevalencia, infecciones oculares parasitarias.

\section{Introducción}

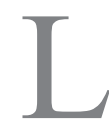

a toxocariosis ocular es una forma de inflamación intraocular debida a la invasión del segmento posterior del ojo por el nematodo Toxocara canis. Constituye una de las causas más frecuentes de uveitis posterior en los niños, pudiendo ocasionar graves complicaciones intraoculares conducentes a la ceguera ${ }^{1-3}$. Se ha reportado una variable prevalencia de seropositividad (prueba serológica inmunoabsorbente ELISA) para $T$. canis. Por ejemplo, en un estudio realizado en Barcelona ${ }^{4}$, incluyendo un gran número de donantes de sangre sanos y pacientes con hipereosinofilia y otras helmintiasis, se estimó 3,6\% de seropositividad. En otro estudio realizado en la República Checa ${ }^{5}$ en población general de distintos distritos, la prevalencia varió entre 5,8 y $36 \%$, siendo más alta en las áreas rurales. No obstante, los datos extraídos de la literatura internacional indican que existe una muy variable prevalencia de lesiones oculares en los niños con serología positiva para T. canis, que podría fluctuar entre $0 \%$ en niños asintomáticos de kindergarten ${ }^{6}$ y $27,7 \%$ en niños con diagnóstico presuntivo de toxocariosis ${ }^{7}$.

Chile es un país con alta prevalencia general de títulos positivos para T. canis, estimándose en $8,8 \%{ }^{8}$ en adultos sanos. Esta cifra consideró como positivo los títulos de ELISA $\geq 1: 64$ (valor superior en dos desviaciones estándares al promedio). No obstante, la prevalencia de lesiones oculares en niños asintomáticos con serología positiva para T. canis o con clínica de toxocariosis sistémica es desconocida en nuestro medio. El examen oftalmológico completo a los niños con sospecha de esta parasitosis es, por lo tanto, el mejor método para identificar las lesiones sugerentes de toxocariosis ocular, debiendo realizarse bajo anestesia general en casos de niños pequeños o poco cooperadores. La estimación de la frecuencia de patología oftalmológica en niños con manifestaciones clínicas de toxocariosis sistémica y/o seropositivos para la enfermedad podría ser de gran utilidad en la elaboración de un algoritmo para examen y seguimiento oftalmológico a fin de maximizar los recursos humanos y el tiempo invertido en su atención hospitalaria.

\section{Objetivo}

Estimar la prevalencia y características clínicas de las lesiones oculares en niños con serología positiva para $T$. canis referidos desde la Unidad de Parasitología del Hospital Luis Calvo Mackenna (HLCM), hospital pediátrico público y de alta complejidad.

\section{Pacientes y Métodos}

Se realizó un estudio retrospectivo de las fichas clínicas de todos los niños con diagnóstico de toxocariosis clínica y/o serológica (títulos $\geq 1: 64$ determinado por técnica de ELISA) derivados a la Unidad de Oftalmología desde la
Hospital Luis Calvo Mackenna, Santiago, Chile. Unidad de Oftalmología (JEST, JPLG, MGN, EVD, DMM, ARB). Unidad de Parasitología (INH, AVA)

Los autores no tienen conflicto de interés con los métodos y/o pacientes que aparecen en el presente estudio.

Recibido: 10 de diciembre de 2010 Aceptado: 11 de mayo de 2011

Correspondencia a: Juan Pablo López G. jpablolopezg@hotmail.com 
Unidad de Parasitología del HLCM para examen oftalmológico entre marzo del año 2005 y agosto del 2009. Se consignó para cada paciente datos demográficos, la presencia o ausencia y el tipo de síntomas y signos oculares al momento del examen y los hallazgos encontrados al examen oftalmológico sugerentes de toxocariosis, incluyendo signos oculares de uveitis y sus secuelas $(\mathrm{Ej}$. cataratas, sinequias posteriores, glaucoma) y la presencia de granulomas retinales característicos de T. canis. La Figura 1 muestra un granuloma retinal característico de toxocariosis de un paciente no incluido en el presente estudio. Los datos se tabularon y analizaron en una planilla Excel ${ }^{\mathrm{TM}}$. Para la determinación de porcentajes, se calculó un intervalo de confianza, utilizando el confidence interval for proportion calculator (disponible en http://dimensionresearch.com/). El estudio contó con la aprobación del Comité de Ética del HLCM.

\section{Resultados}

En el período estudiado se identificaron 175 niños con serología positiva para T. canis, correspondiendo 108 de ellos $(63,5 \%)$ a sexo masculino. La relación de seropositividad de niños/niñas fue de 1,7/1. La edad promedio y la desviación estándar al momento del examen fue de 6 (rango 0,66-15) \pm 4 años, respectivamente, no encontrándose diferencias por sexo. Los títulos serológicos fluctuaron entre 1:64 y 1:1.024. En sólo un niño [(0,57\%); 95\% IC, -0,55-1,69] se encontraron lesiones clínicamente compatibles con compromiso intraocular debido a T. canis. Este niño de 7 años de edad y títulos ELISA de 1: 512 presentaba un granuloma retinal periférico característico de toxocariosis en uno de sus ojos y además disminución de visión (20/40) en su ojo comprometido. Adicionalmente, como hallazgo de examen se identificaron otras patologías oculares en tres niños, presentando dos de ellos síntomas visuales: dos niños $(1,14 \%)$ presentaban lesiones retinales sugerentes de toxoplasmosis ocular y un niño $(0,57 \%)$ tenía hipoplasia del nervio óptico.

Figura 1. Granuloma del área macular en un niño con toxocariosis ocular (ojo izquierdo). El granuloma (blanco) está conectado al nervio óptico mediante un tallo fibroso y genera varios pliegues finos de tracción retinal con deformación de la anatomía retinal. El paciente presenta leucocoria (brillo blanco en la pupila) y estrabismo. Fotografía gentileza de la Dra. Militza González N.

\section{Discusión}

Las infecciones parasitarias del ojo son una causa importante de ceguera en el mundo, siendo la toxocariosis una de las etiologías más frecuentes en Estados Unidos de América, Sudamérica, Canadá y Europa ${ }^{1}$. Algunos estudios indican que la toxocariosis ocular daría cuenta de $37 \%$ del total de la patología retinal pediátrica ${ }^{2}$, en tanto que Toxocara sp y otras larvas migrantes viscerales representarían $28,3 \%$ de todos los casos de uveítis y $9,4 \%$ de todas las uveítis pediátricas ${ }^{3}$. La toxocariosis ocular también se ha reportado en adultos jóvenes y en individuos hasta la edad de 50 años ${ }^{9}$. En nuestro país, un estudio realizado en el Departamento de Úvea del Hospital Del Salvador (centro de referencia nacional de uveítis a nivel público) destacó a la toxocariosis como la causa más frecuente de uveítis posterior encontrada en los niños referidos a dicha unidad (comunicación personal, López JP. et al. Perfil de Morbilidad del Dpto. de Úvea del Hospital Del Salvador, presentado en el XVIII Congreso Chileno de Oftalmología, Pucón 2001). La gravedad de la enfermedad en el humano depende de la carga parasitaria, el sitio de infección, el comportamiento migratorio del parásito y la respuesta inflamatoria del hospedero. La infección puede adoptar la forma de larva migrans visceral o bien de toxocariosis ocular. En el primer caso, la infección se presenta en niños de entre 2 y 4 años con fiebre, síntomas respiratorios, hepato-esplenomegalia y marcada eosinofilia ${ }^{10}$. Aproximadamente $5 \%$ presenta alguna evidencia de compromiso ocular ${ }^{11}$. La toxocariosis ocular, en cambio, se presenta en niños mayores, con un promedio de edad entre 7,5 y 8,6 años $^{11,12}$ y no presenta eosinofilia.

Los pacientes con compromiso intraocular debido a T. canis se presentan con disminución de visión unilateral indolora, estrabismo o leucocoria y la enfermedad bilateral es extremadamente inhabitual ${ }^{13}$. El compromiso ocular es principalmente posterior, adoptando las formas de granuloma periférico, granuloma del polo posterior, endoftalmitis crónica y en otras presentaciones atípicas. En un paciente con manifestaciones clínicas sugerentes, la prueba serológica inmunoabsorbente ELISA para $T$. canis es altamente sensible y específica para detectar exposición previa al microorganismo. El límite de corte de 1:8 otorga un $91 \%$ de sensibilidad y $90 \%$ de especificidad cuando se combina con la expresión clínica ${ }^{14}$, teniendo presente que en enfermedad sistémica se considera con valor diagnóstico un título $\geq 1 / 64$ como fuera expresado en la Introducción. Los títulos decaen en el tiempo, por lo que se ha sugerido que cualquier título positivo podría considerarse significativo en el contexto clínico apropiado. No obstante, existe escasa información en la literatura médica sobre el valor que cobra este examen serológico en pacientes pediátricos asintomáticos visuales que resultan seropositivos para $T$. canis. 
Los oftalmólogos se ven enfrentados en la práctica diaria con dos situaciones clínicas relacionadas con la toxocariosis: el escenario -menos frecuente- de un niño con síntomas y signos oftalmológicos característicos -mala visión, estrabismo, leucocoria, etc-que llevan a su familia a buscar atención oftalmológica, identificándose en su examen de fondo de ojos lesiones características de T. canis y cuyo posterior estudio serológico arroja valores positivos. En un segundo escenario clínico -mucho más común-, un niño asintomático con manifestaciones clínicas sugerentes de toxocariosis sistémica es estudiado con exámenes de laboratorio, constatándose su seropositividad para T. canis y requiere, por lo tanto, examen oftalmológico para descartar el eventual compromiso intraocular. La baja prevalencia de lesiones oculares encontrada en nuestros pacientes hospitalarios con títulos positivos para T. canis sugiere que el rendimiento del examen oftalmológico es bajo en este segundo tipo de pacientes en cuanto a la identificación de lesiones compatibles con toxocariosis, siendo de mayor utilidad si el niño presenta síntomas y/o signos oftalmológicos. Sugerimos que el médico clínico que se vea enfrentado a este tipo de niños seropositivos para T. canis investigue dirigidamente la presencia de mala visión, estrabismo o leucocoria que apoyan fuertemente la posibilidad de compromiso intraocular concomitante.

Para nuestro conocimiento, este es el estudio más grande sobre prevalencia de lesiones oculares en niños con serología positiva para $T$. canis realizado en nuestro país. En un estudio previo realizado en el $\mathrm{HLCM}^{15}$ en 42 niños con síndrome de larva migrans visceral confirmada serológicamente, se encontró alteración del fondo de ojos sugerente de $T$. canis en dos de ellos (4,5\%). La gran mayoría de los pacientes examinados en nuestro estudio estaban asintomáticos o bien habían ya sido tratados con antiparasitarios, por lo que no podemos descartar que una mayor prevalencia de lesiones oculares pueda presentarse durante el cuadro clínico sistémico activo o bien, por el contrario, que el tratamiento antiparasitario tenga algún efecto protector en la invasión intraocular por el parásito. Un estudio prospectivo sobre prevalencia de lesiones oculares antes y después del tratamiento antiparasitario podría ayudar a dilucidar este aspecto.

Nuestro estudio posee varias e importantes limitaciones. Por tratarse de una revisión y no contar con un grupo control, no puede descartarse el sesgo propio de este tipo de diseños experimentales. La muestra estudiada fue altamente seleccionada, incluyendo pacientes con clínica de toxocariosis sistémica atendidos en una unidad de parasitología de un hospital terciario y no constituye, por lo tanto, un índice orientador de la prevalencia de lesiones oculares en niños seropositivos en la población general. La realidad hospitalaria difiere grandemente de la situación de atención de los niños en la atención primaria, donde probablemente la prevalencia de serología positiva para toxocariosis es también alta en pacientes asintomáticos (cuya prevalencia de lesiones oculares es desconocida), por lo que la generalización de nuestros resultados debe hacerse con cautela.

Por último, los niños pequeños generalmente no refieren sus síntomas visuales, por lo que puede presentarse la situación de encontrar lesiones oculares sugerentes de toxocariosis en un niño totalmente asintomático.

Dada la muy baja prevalencia $(0,57 \%)$ de lesiones oculares en niños seropositivos y asintomáticos visuales encontrada en nuestra serie, esperamos que la información obtenida en este estudio pueda ser de utilidad en el diseño de un algoritmo de orientación y enfoque más específico del examen oftalmológico orientado a niños con laboratorio compatible con toxocariosis en el ambiente hospitalario, donde el recurso oftalmológico para atención pediátrica es reconocidamente bajo a nivel nacional.

\section{Resumen}

La invasión intraocular por el nematodo Toxocara canis constituye una de las causas más frecuentes de uveitis en los niños, pudiendo conducir a la ceguera. Para conocer la prevalencia de lesiones oculares en niños con serología positiva para este parásito en un hospital pediátrico de referencia, realizamos un estudio retrospectivo de las fichas clínicas de los pacientes referidos para examen oftalmológico en el Hospital Luis Calvo Mackenna entre los años 2005 y 2009. Encontramos 175 niños con serología positiva y examen oftalmológico, cuya edad promedio al momento del examen fue de 6 años (rango $0,66-15)$. En sólo un niño [(0,57\%); 95\% IC, -0,55-1,69] se encontraron lesiones oculares atribuibles a $T$. canis. El paciente presentaba además disminución de visión. La información obtenida en este estudio podría ser de utilidad en el diseño de un algoritmo para el examen oftalmológico de niños seropositivos a $T$. canis en el ambiente hospitalario en Chile.

\section{Referencias}

1.- Rubinsky-Elefant G, Hirata C E, Yamamoto J H, Ferreira M U. Human toxocariasis: diagnosis, worldwide seroprevalences and clinical expression of the systemic and ocular forms. Ann Trop Med Parasitol 2010; 104 (1): 3-23.
2.- Schantz P M, Weis P E, Pollard Z F, White M C. Risk factors for toxocaral ocular larva migrans: a case-control study. Am J Public Health 1980; 70 (12): 1269-72.

3.- Benezra D, Cohen E, Maftzir G. Patterns of intraocular inflammation in children. Bull Soc Belge Ophtalmol 2001; (279): 35-38.
4.- Portús M, Riera C, Prats G. A serological survey of toxocariasis in patients and healthy donors in Barcelona (Spain). Eur J Epidemiol 1989; 5 (2): 224-7.

5.- Uhlíková M, Hübner J. Seroprevalence of Toxocara canis infection in Czech Republic. Cent Eur J Public Health 1998; 6 (3): 195-8. 
6.- Ellis G S, Pakalnis V A, Worley G, Green J A, Frothingham T E, Sturner R A, et al. Toxocara canis infestation. Clinical and epidemiological associations with seropositivity in kindergarten children. Ophthalmology 1986; 93 (8): 1032-7.

7.- Altcheh J, Nallar M, Conca M, Biancardi M, Freilij H. Toxocariasis: clinical and laboratory features in 54 patients. An Pediatr (Barc) 2003; 58 (5): 425-31.

8.- Hersković P, Astorga B. Human toxocariasis in Chile. Rev Med Chile 1985; 113 (1): 18-21.

9.- Raistrick E R, Hart J C. Adult toxocaral infection with focal retinal lesion. Br Med J 1975; 3 (5980): 416.

10.- Schantz P M, Glickman L T. Toxocaral visceral larva migrans. N Engl J Med 1978; 298 (8): 436-9.

11.- Brown D H. Ocular Toxocara canis II. Clinical review. J Pediatr Ophthalmol 1970; 7: 182-91.

12.- Schantz P M, Meyer D, Glickman L T. Clinical, serologic, and epidemiologic characteristics of ocular toxocariasis. Am J Trop Med Hyg 1979; 28 (1): 24-8.

13.- Benítez del Castillo JM, Herreros G, Guillen JL,
Fenoy S, Bañares A, Garcia J. Bilateral ocular toxocariasis demonstrated by aqueous humor enzyme-linked immunosorbent assay. Am J Ophthalmol 1995; 119 (4): 514-6.

14.- Pollard Z F, Jarrett W H, Hagler W S, Allain D S, Schantz P M. ELISA for diagnosis of ocular toxocariasis. Ophthalmology 1979; 86 (5): 743-52.

15.- Noemí H I, Schuh O W, Herskovic L P, Ríos L E, Cerva C L, Torres C MT, et al. Larva migrans visceral en niños. Rev Chil Pediatr 1984; 55 (4): 244-8. 